Article

\title{
Extraction of Partial Discharge Pulses from the Complex Noisy Signals of Power Cables Based on CEEMDAN and Wavelet Packet
}

\author{
Kang Sun ${ }^{1, *}$, Jing Zhang ${ }^{1}$, Wenwen Shi ${ }^{2}$ and Jingdie Guo ${ }^{3}$ \\ 1 School of Electrical Engineering and Automation, Henan Polytechnic University, Jiaozuo 454000, China \\ 2 China Aluminum Logistics Group Zhongzhou Co., Ltd., Xinxiang 453000, China \\ 3 State Grid Jiyuan power supply company, Jiyuan 459000, China \\ * Correspondence: sunkang@hpu.edu.cn; Tel.: +86-152-3904-3197
}

Received: 17 July 2019; Accepted: 20 August 2019; Published: 22 August 2019

\begin{abstract}
While both periodic narrowband noise and white noise are significant sources of interference in the detection and localization of partial discharge (PD) signals in power cables, existing research has focused nearly exclusively on white noise suppression. This paper addresses this issue by proposing a new signal extraction method for effectively detecting random PD signals in power cables subject to complex noise environments involving both white noise and periodic narrowband noise. Firstly, the power cable signal was decomposed using complete ensemble empirical mode decomposition with adaptive noise (CEEMDAN), and the periodic narrowband noise and frequency aliasing in the obtained signal components were suppressed using singular value decomposition. Then, signal components contributing significantly to the PD signal were determined according to the cross-correlation coefficient between each component and the original PD signal, and the PD signal was reconstructed solely from the obtained significant components. Finally, the wavelet packet threshold method was used to filter out residual white noise in the reconstructed PD signal. The performance of the proposed algorithm was demonstrated by its application to synthesized PD signals with complex noise environments composed of both Gaussian white noise and periodic narrowband noise. In addition, the time-varying kurtosis method was demonstrated to accurately determine the PD signal arrival time when applied to PD signals extracted by the proposed method from synthesized signals in complex noise environments with signal-to-noise ratio (SNR) values as low as $-6 \mathrm{~dB}$. When the SNR was reduced to $-23 \mathrm{~dB}$, the arrival time error of the PD signal was only one sampling point.
\end{abstract}

Keywords: power cables; denoising; complete ensemble empirical mode decomposition with adaptive noise; singular value decomposition; signal-to-noise ratio

\section{Introduction}

The electrical insulation of high-voltage power cables is subject to stresses that can result in local dielectric breakdowns that are typically preceded by partial discharge (PD) pulse signals. As such, the online detection of the PD signals of power cables can provide information regarding the locations of weak points or line faults in power distribution systems according to the characteristics of the PD signals obtained at monitoring points, such as arrival times or received signal strengths, and thereby provides an effective means for ensuring the economic and reliable operation of power systems via timely repair [1-4]. In recent years, the PD pulse signals of line faults have been commonly detected by coupling power cable signals with a high-frequency current sensor (HFCT). However, PD signals are convoluted with a relatively high level of background noise of various types, which results in a 
complex overall power cable signal with a low signal-to-noise ratio (SNR) relative to the PD signal of interest. This greatly complicates the task of fault localization.

Existing fault localization algorithms can be classified according to the various means by which line distances are measured. Fault localization without distance measurements is mainly realized by communication technology and the localization accuracy is poor. In contrast, localization algorithms employing distance measurements are based on various PD signal features, such as time difference of arrival (TDOA) [5], time of arrival (TOA) [6], angle of arrival (AOA) [7], or received signal strength indicator (RSSI) [8]. However, the fault localization accuracy of each of these methods suffers from various limitations associated with the challenging characteristics of PD signals. For example, the partial discharge location method based on AOA [7] and signal RSSI [8] does not consider the noise in the actual environment. The fault localization accuracies of algorithms based on the arrival time difference threshold method [9] and the half-wave-based cross-correlation method [10] are limited by the SNR of the PD signal. The binary mapping method based on the continuous wavelet transform (CTW) converts the CTW coefficient into a binary grayscale image and takes the first non-zero pixel as the wave arrival time. This method is also affected by the noise in the signal [11]. The cross-correlation algorithm based on a half-wavefront was proposed and used to locate the PD source in air-insulated substations, and the half-wavefront extraction result was affected by the SNR [10]. Finally, the localization method based on wavelet packet kurtosis [12] considered only PD signals containing white noise. Therefore, developing methods for extracting low SNR PD signals under complex background noise is essential for ensuring high fault-localization accuracy.

The main types of background noise can be divided into three categories, including white noise, periodic narrowband noise, and impulse interference. The wide-frequency distribution of white noise generally overlaps with the frequency bands of PD signals, making white noise difficult to suppress completely. Periodic narrowband noise is relatively common and has a high degree of interference with PD signals, making it the primary object of noise suppression. In contrast, periodic pulse noise is easily filtered out because of its strong regularity. The most common methods employed for denoising PD signals in recent years include the fast Fourier transform (FFT) [13], wavelet transform [14], and empirical mode decomposition (EMD) [15]. The FFT is a global noise analysis method that can achieve good denoising results for signals that change slowly with time. Denoising methods based on the wavelet transform have often achieved superior denoising results by conducting signal analysis based on a sparse representation of the signal. However, these methods cannot adaptively select the wavelet base and the number of decomposition layers and are therefore limited in their application. In contrast, the EMD-based denoising method realizes the adaptive decomposition of signals with great flexibility. However, this method suffers from the frequency aliasing problem and the endpoint effects remaining after conducting the decomposition cannot be suppressed, which is particularly problematic when attempting to select signal components that make a significant contribution to the PD signal, because significant components that are not readily detectable can be easily omitted, and thus, negatively impact subsequent signal analysis.

This paper addresses the above-discussed challenges by proposing a signal extraction algorithm based on complete ensemble EMD with adaptive noise (CEEMDAN) to detect significant signal components and suppress periodic narrowband noise. Wavelet thresholding is employed to filter out the residual white noise in the significant signal components detected by CEEMDAN. In Section 2, the decomposition performance of the CEEMDAN algorithm is analyzed. In addition, we address the frequency aliasing problem and limit the influence of interference derived from periodic narrowband noise using singular value decomposition (SVD). In Section 3, the performances of existing wavelet threshold functions for suppressing white noise are analyzed and a new threshold function is proposed that can effectively address the disadvantages of applying conventional soft and hard thresholds. Then, the overall processing flow of the proposed PD signal extraction algorithm is briefly summarized in Section 4. In Section 5, the extraction results of the partial discharge signals of the algorithm described in this paper are compared with those of other existing signal extraction algorithms, and the algorithm 
is applied to determine the arrival times of the measured PD signals with different SNR values. Finally, the conclusions of the work are presented in Section 6.

\section{PD Signal Extraction Based on the CEEMDAN Algorithm}

\subsection{CEEMDAN Algorithm}

The EMD algorithm decomposes time-based signals into finite sets of mono-components with different amplitude and frequency bands. These components are denoted as intrinsic mode functions (IMFs). The discussed problems associated with frequency aliasing, false components, and the endpoint effects of EMD were addressed by Lei et al. [16] by proposing ensemble EMD (EEMD). The EEMD algorithm adds a known level of white noise to a signal and then decomposes it by EMD. This process is repeated $n$ times with different white noise levels and the final signal components are obtained as the average values of the $n$-resulting IMFs. However, the presence of residual white noise induces errors in the reconstructed signals. This issue was addressed by Yeh et al. [17] by proposing a complete EEMD (CEEMD), which reduces the residual white noise in the reconstructed signal by adding both positive and negative noise in pairs prior to conducting EEMD. However, the calculation burden associated with the decomposition process is large and the reconstructed signals tend to be incomplete. In contrast with these earlier EMD approaches, CEEMDAN [18] adaptively adds white noise to the original signal. This approach not only solves the problem of obtaining different numbers of IMFs after adding different white noise levels, but also reduces the calculation burden of the CEEMD process, and thereby obtaines relatively complete reconstructed signals.

The CEEMDAN process is similar to that of EEMD for the first IMF. Here, $n$ times of different white noise levels $s_{i}(t), i=1,2,3, \ldots, n$, were added into the original noise-containing signal $y(t)$, and EMD was conducted to obtain the first $\operatorname{IMF}\left(F_{1}\right)$ for each noise level. The mean value of $F_{1}$ is the first $\operatorname{IMF}\left(I M F_{1}\right)$ of CEEMDAN as follows:

$$
I M F_{1}=\frac{1}{n} \sum_{i=1}^{n}\left(F_{1}\left(y(t)+s_{i}(t)\right)\right)
$$

Then, $I M F_{1}$ is removed from $y(t)$ to obtain the first residual signal as follows:

$$
M_{1}(t)=y(t)-I M F_{1} .
$$

The process is repeated with $M_{1}$ and new white noise levels $\sigma_{1} F_{1}\left(s_{i}(t)\right)$ to obtain $I M F_{2}$ :

$$
I M F_{2}=\frac{1}{n} \sum_{i=1}^{n} F_{1}\left(M_{1}(t)+\sigma_{1} F_{1}\left(s_{i}(t)\right)\right),
$$

where $\sigma_{1}$ is the noise coefficient. In this paper, $\sigma_{k}$ was set as the standard deviation of $M_{k}(t), k=1,2,3$, $\ldots, K$, where $K$ is the total number of IMFs obtained in the decomposition process. By analogy, the $k$ th residual signal for $k>1$ is:

$$
M_{k}=M_{k-1}(t)-I M F_{k}
$$

and the $k$ th IMF is given as:

$$
I M F_{k}=\frac{1}{n} \sum_{i=1}^{n} F_{k}\left(M_{k}(t)+\sigma_{k} F_{k}\left(s_{i}(t)\right)\right) .
$$

The CEEMDAN process is stopped when $M_{K}(t)$ is a monotonic signal, and $y(t)$ can be expressed as:

$$
y(t)=\sum_{k=1}^{K} I M F_{k}+M_{K}(t) .
$$


where $F_{k}(\cdot)$ represents the $k$ th IMF decomposed by EMD. $\sigma_{k}$ is the noise figure. In this paper, the noise figure was determined by calculating the standard deviation of the residual component $M_{k}(t)$.

\subsection{Effective Selection of Significant IMFs}

The key to ensuring the accurate extraction of signals based on the EMD algorithm is to select IMFs from the decomposition results that are significantly representative of PD signals. Currently, the effective selection of significant IMFs relies mainly on the autocorrelation coefficient [19], the energy threshold [20], and the kurtosis value [21]. However, these methods for selecting IMFs may fail to detect significant components or incorrectly select irrelevant components when the IMF contains a high proportion of narrowband noise.

We investigated this issue by applying SVD to representative PD signals with various types of added noise, including an original PD signal, a PD signal containing Gaussian white noise, and a PD signal containing periodic narrowband noise. SVD generally considers that only the first $q$ large singular values among the non-zero singular values obtained by the decomposition process correspond to significant signal components and the remaining singular values are considered redundant components, where $q$ represents the number of significant components. Therefore, only the first $q$ singular values were selected for signal reconstruction to achieve signal denoising. However, the singular value characteristics corresponding to the PD signals containing Gaussian white noise and periodic narrowband noise differed considerably, as shown in Figure 1, which considers only the first 30 singular values. The figure indicates that the singular values of the three signals were essentially equivalent, except that the PD signal with periodic narrowband noise spiked in the first few singular values. According to this feature, the present study employed the results of SVD to suppress periodic narrowband noise in the components obtained by CEEMDAN.

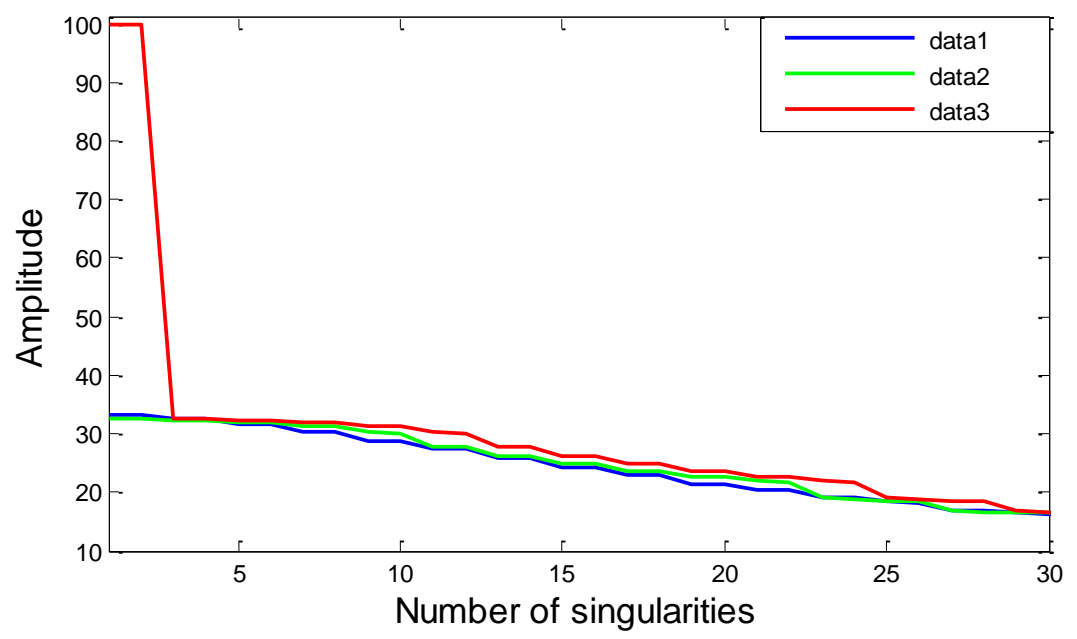

Figure 1. Characteristic curves of the singular values of an original partial discharge (PD) signal (data 1); the PD signal with added Gaussian white noise (data 2); and the PD signal with added periodic narrowband noise (data 3).

In order to better perform SVD, we constructed the following Hankel matrix for each $\operatorname{IMF}=\left(x_{1}\right.$, $\left.x_{2}, \ldots, x_{n}\right)$ obtained in the CEEMDAN process [22]:

$$
H_{k}=\left(\begin{array}{ccccc}
x_{1} & x_{2} & x_{3} & \cdots & x_{m} \\
x_{2} & x_{3} & x_{4} & \cdots & x_{m+1} \\
x_{3} & x_{4} & x_{5} & \cdots & x_{m+2} \\
\vdots & \vdots & \vdots & \ddots & \vdots \\
x_{n-m+1} & x_{n-m+2} & x_{n-m+3} & \cdots & x_{n}
\end{array}\right)
$$


where $1<m<n$. We then performed SVD processing on $H_{k}$, and the form of the decomposition is shown as Equation (8):

$$
H_{k}=U D V^{T}
$$

where $U$ is an $m \times m$ orthogonal matrix, $D$ is an $m \times n$ diagonal matrix, and $V$ is an $n \times n$ orthogonal matrix. The first few singular values characteristic of periodic narrowband noise were set to zero as follows:

$$
D_{i i}=\left\{\begin{array}{c}
D_{i i}, D_{i i}-D_{i-1 i-1} \leq \alpha \times\left(D_{i-1 i-1}-D_{i-2 i-2}\right) \\
0, D_{i i}-D_{i-1 i-1}>\alpha \times\left(D_{i-1 i-1}-D_{i-2 i-2}\right)
\end{array} .\right.
$$

Here, the function $\alpha$ is a constant. Then, the inverse SVD operation was applied to the resulting $H_{k}$ to obtain $I M F_{k}$ with the periodic narrowband noise removed. The PD signal was then reconstructed from the set of IMFs with the periodic narrowband noise removed.

This paper introduced the cross-correlation method for selecting significant IMFs. The cross-correlation method is used in the field of signal processing to represent the similarity between two signals, where one is a known signal and the other an unknown signal, and the characteristics of the unknown signal are determined according to its similarities with known signals [18]. In general, the cross-correlation coefficient $\rho$ between a PD signal $f(t)$ and noise is very small. Therefore, the value of $\rho$ between $f(t)$ and a given $I M F_{k}$ increases at the extent to which $I M F_{k}$ represents the PD signal increases. In addition, the magnitude of $\rho$ is independent of the signal amplitude. Therefore, a significant IMF was determined according to the value of $\rho$, which is calculated as follows:

$$
\rho=\frac{\sum_{i=1}^{l}(f(i)-\bar{f})\left(I M F_{k}(i)-\overline{I M F}_{k}\right)}{\sqrt{\sum_{i=1}^{l}(f(i)-\bar{f})^{2}} \sqrt{\sum_{i=1}^{l}\left(I M F_{k}(i)-\overline{I M F_{k}}\right)^{2}}} .
$$

where $l$ is the length of $I M F_{k}$, and $\bar{f}$ and $\overline{I M F}$ are the average values of $f(i)$ and $I M F_{k}(i)$, respectively. The PD signal was then reconstructed from the set of significant IMFs.

\section{Adaptive Wavelet Packet Threshold Method}

The selection of the threshold function in the wavelet packet threshold denoising process is a key step. The application of traditional soft and hard threshold function is limited by its discontinuity and constant error. To this end, many improved threshold functions have been proposed in recent years [23,24]. Three of the more sophisticated threshold functions are given in Equations (11)-(13) below:

$$
\begin{gathered}
\widetilde{d}_{j, k}=\left\{\begin{array}{c}
\operatorname{sign}\left(d_{j, k}\right)\left(\left|d_{j, k}\right|-a \sigma\right),\left|d_{j, k}\right| \geq \sigma \\
0,\left|d_{j, k}\right|<\sigma
\end{array}\right. \\
\widetilde{d}_{j, k}=\left\{\begin{array}{c}
\operatorname{sign}\left(d_{j, k}\right)\left(\left|d_{j, k}\right|-\frac{\sigma}{\left(\left|d_{j, k}\right|^{2}-\sigma^{2}\right)^{j}+1}\right) \\
0,\left|d_{j, k}\right|<\sigma
\end{array},\left|d_{j, k}\right| \geq \sigma\right. \\
\widetilde{d}_{j, k}=\left\{\begin{array}{c}
\operatorname{sign}\left(d_{j, k}\right)\left[\left|d_{j, k}\right|-\sin \left(\frac{\pi}{2}\left|\frac{\sigma}{d_{i, j}}\right|^{z}\right) \sigma\right],\left|d_{j, k}\right| \geq \sigma \\
0,\left|d_{j, k}\right|<\sigma
\end{array}\right.
\end{gathered}
$$

Here, $d_{j, k}$ represents the $k$ th wavelet coefficient of the $j$ th layer in the wavelet packet decomposition, $\left|d_{j, k}\right|$ is the absolute value of $d_{j, k}, \operatorname{sign}(\cdot)$ is the sign function that obtains the sign of $d_{j, k}, a$ is an adjustment coefficient $(0<a<1), z$ is a constant, and $\sigma$ is the threshold, which is calculated as follows:

$$
\sigma=\operatorname{median}\left(\left|d_{j, k}\right|\right) \times \sqrt{2 \ln N} / 0.6745,
$$


where the function median $(\cdot)$ obtains the median value of $\left|d_{j, k}\right|$ and $N$ is the length of $d$. Equation (11) adjusts the constant deviation between the wavelet coefficient of the noisy signal and the estimated noise-free wavelet coefficient of the soft threshold function by adjusting the coefficient $a$. Equation (12) introduces the wavelet decomposition layer number $j$ as an adjustment parameter to reduce the constant deviation that exists in the function. Finally, Equation (13) introduces a sine function for 'smooth' processing in order to bring $\widetilde{d}_{j, k}$ closer to $d_{j, k}$ and adjusts the degree of approximation by parameter z. However, although the above functions ensure continuity, they do not fundamentally solve the constant deviation. Based on this analysis, this paper proposed the following threshold function:

$$
\begin{gathered}
\widetilde{d}_{j, k}=\left\{\begin{array}{c}
(1-b) \operatorname{sign}\left(d_{j, k}\right) \times\left(\left|d_{j, k}\right|-\mu \sigma\right)+b d_{j, k},\left|d_{j, k}\right| \geq \sigma_{1} \\
0,\left|d_{j, k}\right|<\sigma_{1}
\end{array}\right. \\
\text { Here, } \sigma_{1}=\operatorname{median}\left(\left(\left|d_{j, k}\right|-\operatorname{median}\left(d_{j, k}\right)\right)\right) \times \sqrt{2 \ln N} / 0.6745, \\
b=1-\exp \left(-u \times \frac{\left|d_{j, k}\right|-\sigma_{1}}{\left|d_{j, k}\right|+\sigma_{1}}\right), u>0, \text { and } \\
\mu=\exp \left(-m \times \frac{\left|d_{j, k}\right|-\sigma_{1}}{\left|d_{j, k}\right|+\sigma_{1}}\right), m>0 .
\end{gathered}
$$

Here, $u$ and $m$ are adjustment coefficients. In order to reduce the constant deviation of the function itself, the parameter $b$ was introduced to compensate the deviation, and the degree of approximation between $\widetilde{d}_{j, k}$ and $d_{j, k}$ was adjusted by the constants $u$ and $m$. For the convenience of comparison, taking the tangent function as an example, the threshold was set to 0.3 and the processing results of Equations (11)-(13) and (15) are shown in Figure 2.

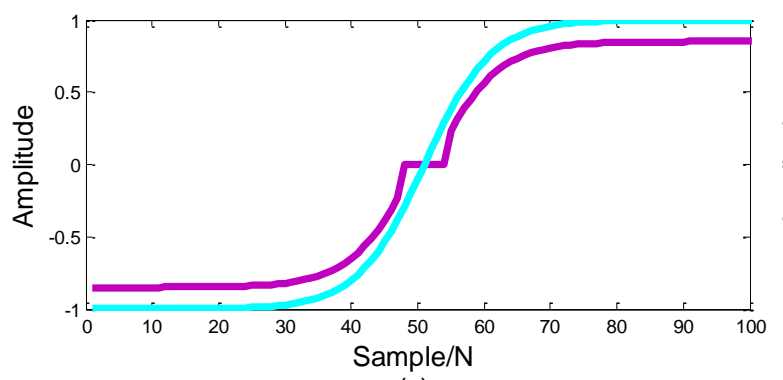

(a)

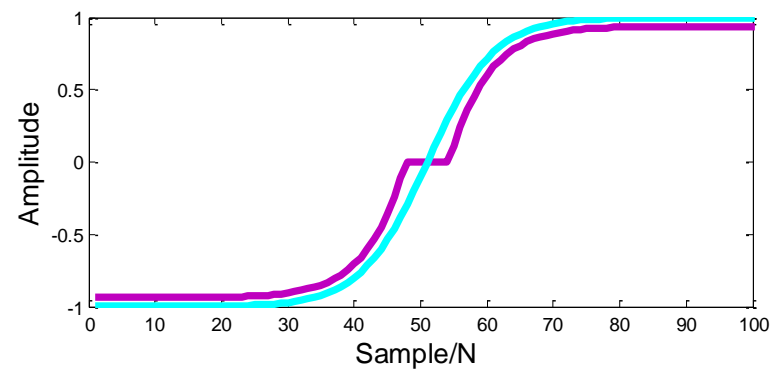

(c)

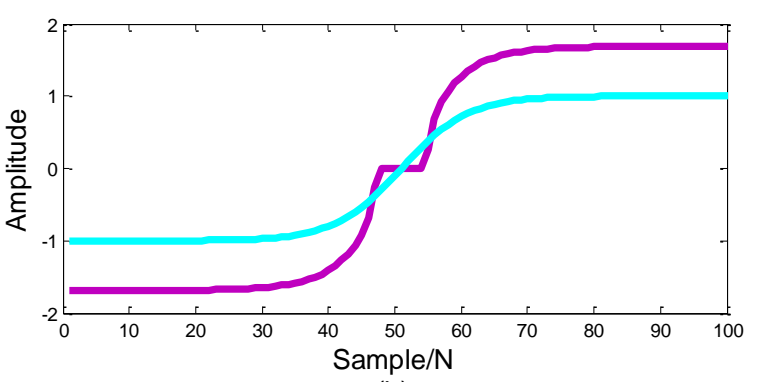

(b)

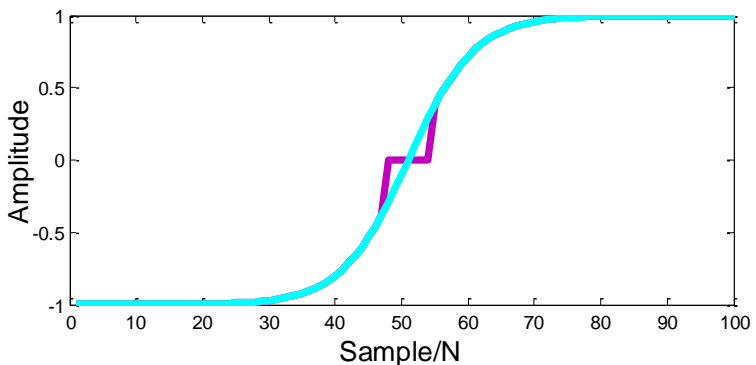

(d)

Figure 2. Comparison of threshold processing results: (a) Threshold function processing result of Equation (11); (b) Threshold function processing result of Equation (12); (c) Threshold function processing result of Equation (13); and (d) Threshold function processing result of Equation (15).

The residual white noise in the reconstructed PD signal was then suppressed using the improved wavelet packet threshold method. 


\section{Signal Extraction Algorithm Based on Combined CEEMDAN-Hankel-SVD}

This paper proposed a PD signal extraction method denoted as the CEEMDAN-Hankel-SVD algorithm that combines CEEMDAN and SVD based on the Hankel matrix formulation as follows:

1. Apply CEEMDAN to a noisy PD signal and obtain $N$ IMFs.

2. Construct a Hankel matrix for each IMF and accordingly apply SVD. The singular values characteristic of periodic narrowband noise are set to zero and the PD signal is reconstructed from the set of IMFs with the periodic narrowband noise removed.

3. Calculate the cross-correlation between each remaining IMF and the original signal and accordingly extract the significant IMFs.

4. The PD signal is finally reconstructed from the significant IMFs and the residual white noise in the reconstructed signal is suppressed using the improved wavelet packet threshold method.

\section{Results and Discussion}

\subsection{PD Signal Simulation}

The noise-free signals $f(t)$ produced by different PD mechanisms for different types of power cables can typically be represented by the following two mathematical models [25].

Single exponential oscillation attenuation model:

$$
f(t)=A e^{-t / \tau} \sin \left(2 \pi f_{c} t\right)
$$

Double exponential oscillation attenuation model:

$$
f(t)=A\left(e^{-1.3 t / \tau}-e^{-2.2 t / \tau}\right) \sin \left(2 \pi f_{c} t\right)
$$

In the above models, $A$ is the amplitude of the signal, $\tau$ is the attenuation coefficient, and $f_{c}$ is the oscillation frequency. In this paper, we applied Equations (16) and (17) as simulated noise-free PD signals, where we employed the parameter $A$ as the normalized amplitude for both simulated signals $\tau=2 \mu \mathrm{s}$ (for Equation (16)), $\tau=1.3 \mu \mathrm{s}$ (for Equation (17)), and $f_{c}=1.2 \mathrm{MHz}$ (for both simulated signals). In addition, we employed a sampling frequency of $20 \mathrm{MHz}$ and a sampling duration of $200 \mu \mathrm{s}$. The waveforms of these two noise-free PD signals are shown in Figure 3a. To these noise-free PD signals, the common noise environment of a power cable involving common white noise and periodic narrowband noise was included. Here, standard Gaussian white noise with a mean of 0 and a variance of 0.1 was adopted, and the periodic narrowband noise was simulated using superimposed sinusoidal signals of $100 \mathrm{kHz}, 500 \mathrm{kHz}, 1.2 \mathrm{MHz}, 5 \mathrm{MHz}$, and $7 \mathrm{MHz}$ with amplitudes of $0.1,0.1,0.3,0.6$, and 1.2, respectively. The resulting noisy PD signal with an SNR of $-6 \mathrm{~dB}$ is shown in Figure $3 \mathrm{~b}$.

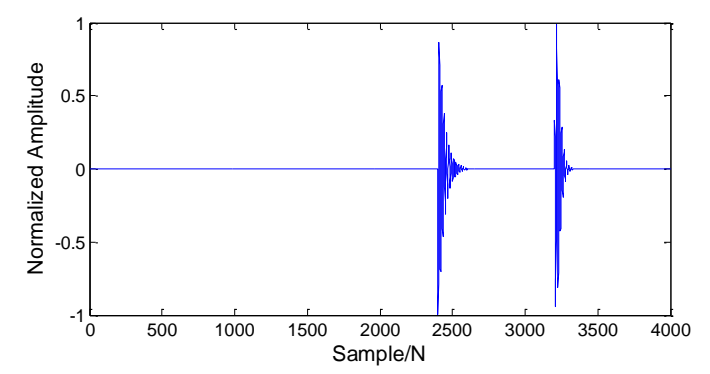

(a)

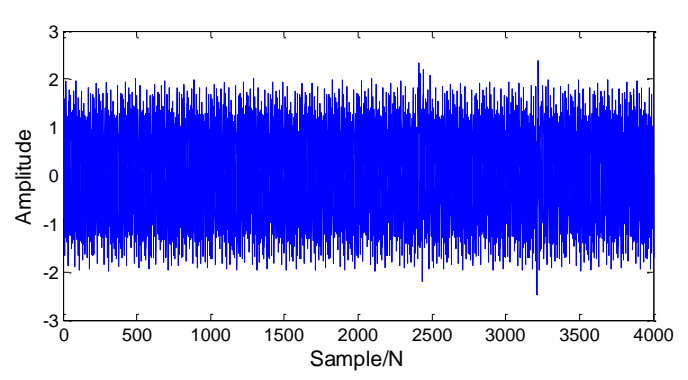

(b)

Figure 3. Simulated PD signal waveforms: (a) Noise-free PD waveform based on (16) and (17); and (b) Noisy PD waveform with Gaussian white noise and periodic narrowband noise providing an signal-to-noise ratio (SNR) of $-6 \mathrm{~dB}$. 


\subsection{PD Signal Extraction and Discussion}

The PD signal shown in Figure $3 b$ was extracted using the proposed CEEMDAN-Hankel-SVD algorithm. The white noise intensity added when solving the first IMF during CEEMDAN processing was 0.2 and the number of cycles was $n=100$. The $12 \mathrm{IMFs}$ obtained during processing are shown in Figure 4, which includes their time-domain waveforms (left) and frequency spectra (right).
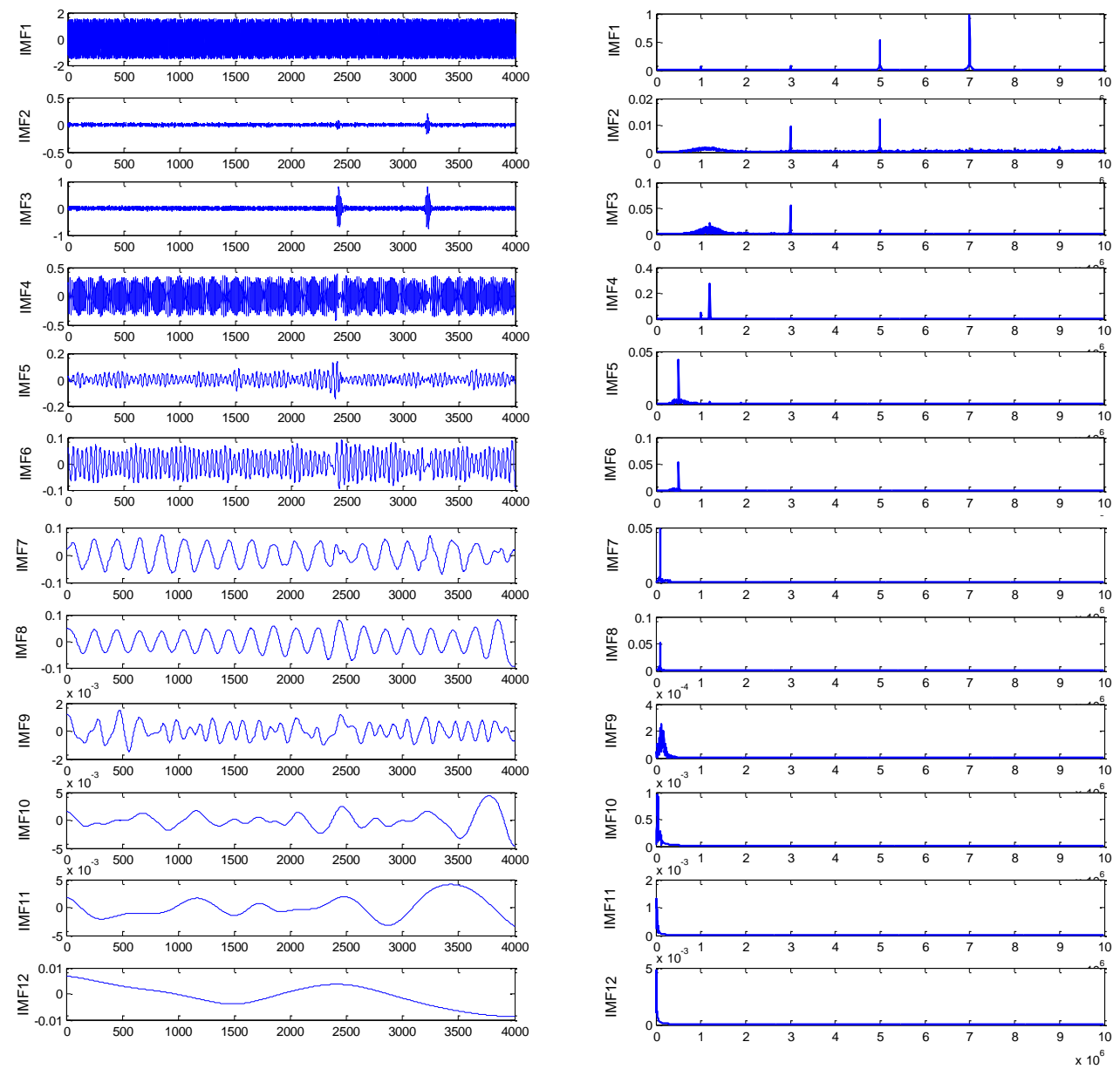

Figure 4. Intrinsic mode functions (IMFs) obtained by complete ensemble empirical mode decomposition with adaptive noise (CEEMDAN): time domain waveforms (left) of each IMFs and its corresponding frequency domain waveform (right).

Intuitively, we noted that the time-domain waveforms of IMF2 and IMF3 were the most obvious IMFs significantly related to the noise-free signals in Figure 3a. However, reconstructing a noise-free PD signal from these two IMFs alone would result in the loss of information. Therefore, the periodic narrowband interference in each IMF component was suppressed by applying SVD with a Hankel matrix formulation, and the value of $\rho$ for each SVD-processed IMF was calculated to select the significant IMFs. A thorough analysis of the experimental data indicates that an IMF can be considered significant when $\rho>0.001$. Figure 5 presents the SVD results for the eight significant IMFs (right), and the results for these IMFs from the right side of Figure 4 were again included for these eight significant IMFs on the left side of Figure 5 to more easily compare the changes in the spectra obtained after suppressing the periodic narrowband noise in the PD signal. 

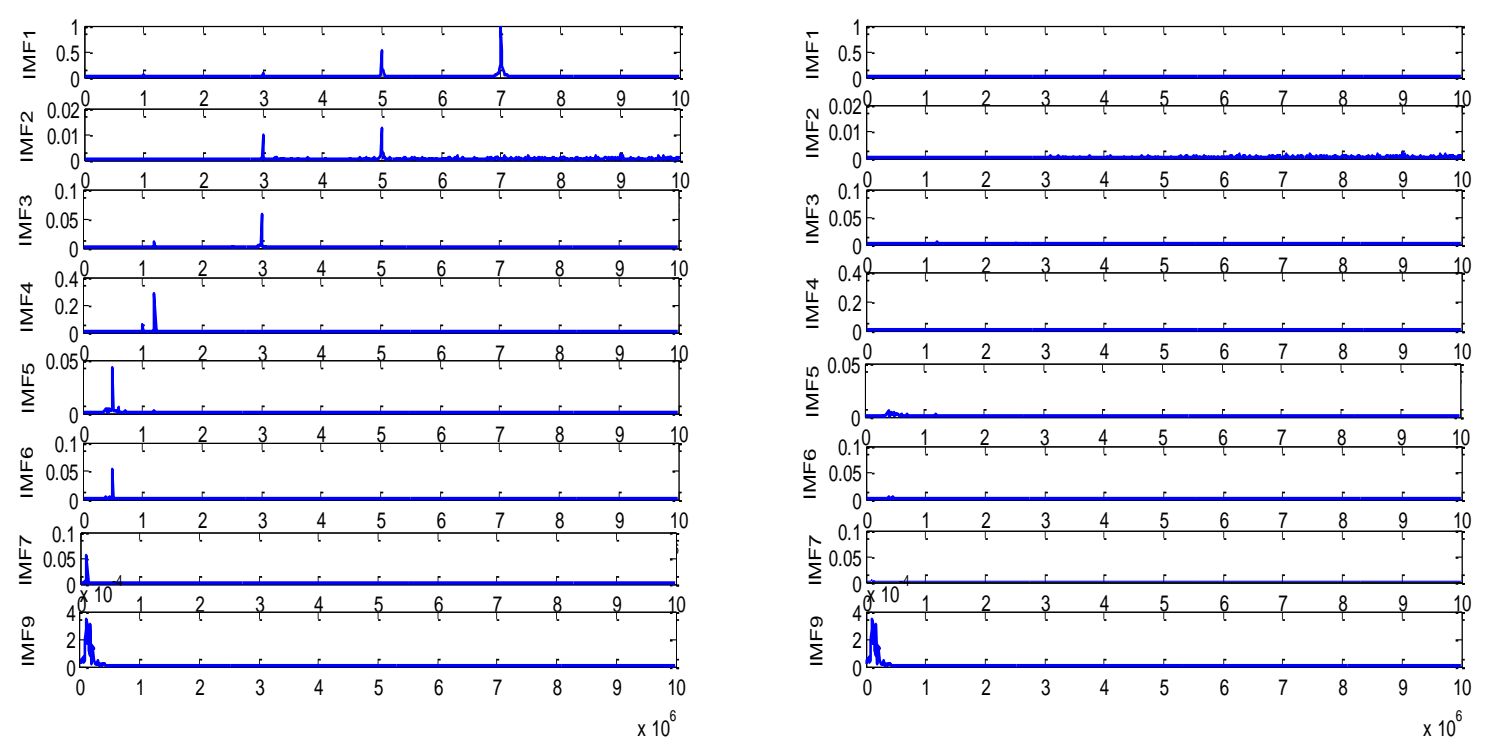

Figure 5. Frequency spectra of the eight significant IMFs detected by the proposed CEEMDAN-Hankel-SVD algorithm before the application of singular value decomposition (SVD) (left; reproduced here from the right side of Figure 3) and after the application of SVD (right).

The results in Figure 4 indicate that, while the periodic narrowband noise was substantially suppressed in the eight significant IMFs, a considerable amount of residual white noise remained in each IMF. This was addressed by employing the $\mathrm{db} 10$ wavelet as the wavelet base in combination with the proposed threshold function (15) to suppress the white noise in the PD signal reconstructed from the significant SVD-processed IMFs, and the resulting waveform is as shown in Figure 6a. The extraction performance of the proposed algorithm was verified by comparison with the extracted signals obtained by the CEEMD-based method [26] and the variation mode decomposition (VMD) wavelet threshold method [27] for the noisy PD signal in Figure 3b. The CEEMD-based method first applies CEEMD to the noisy signal, selects the significant IMFs according to kurtosis values, reconstructs the signal based on the significant IMFs, and obtains the denoised signal by a wavelet hard threshold method. The VMD wavelet threshold method first selects the appropriate decomposition layer to apply VMD to the noisy signal, uses the similarity coefficient to select the significant components, applies threshold denoising to the significant components, and then combines the denoised components and again applies wavelet threshold denoising. The extraction results of the CEEMD-based method and the VMD wavelet threshold method for the simulated PD signal are shown in Figure $6 \mathrm{~b}, \mathrm{c}$, respectively.

The SNR after signal extraction, the normalized correlation coefficient (NCC), the variation trend parameter (VTP), and the amplitude relative error were introduced as evaluation indicators of the extraction performances of the PD signal extraction methods illustrated in Figure 6. These indicators have been defined in detail elsewhere [28]. Here, we noted that the noise suppression ability of a corresponding method increased with an increasing SNR after signal extraction. The NCC employs a value between -1 and 1 to evaluate the similarity between the extracted waveform and the ideal noise-free waveform, where the similarity between the two waveforms increases as the absolute value of the NCC increases from 0 to 1 . The VTP evaluates the degree of similarity between the general trends of two waveforms, where the similarity of the trends increases as the VTP increases from 0 to 1. The amplitude relative error reflects the degree of the energy loss of the signal during the extraction process, where an amplitude relative error value of 0 indicates no energy loss. The results of the four evaluation indexes for the PD signals shown in Figure 6 extracted by the three PD signal extraction methods are listed in Table 1. 


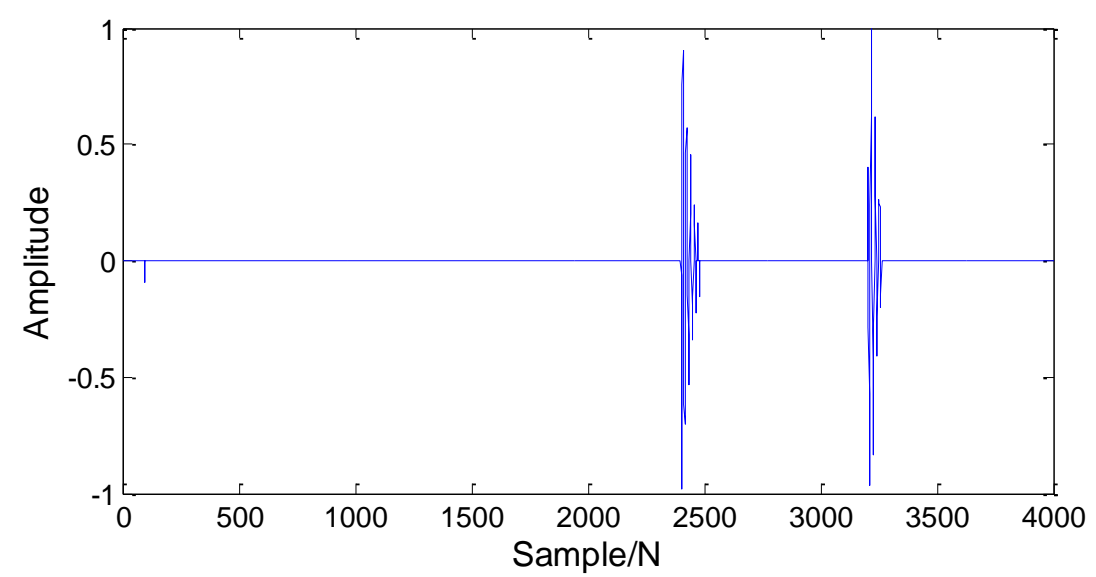

(a)

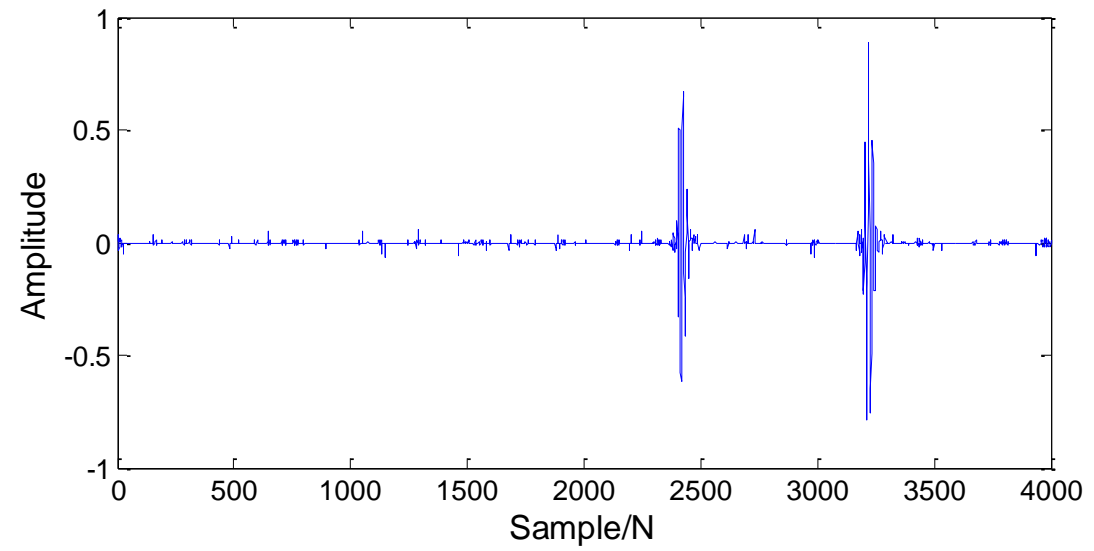

(b)

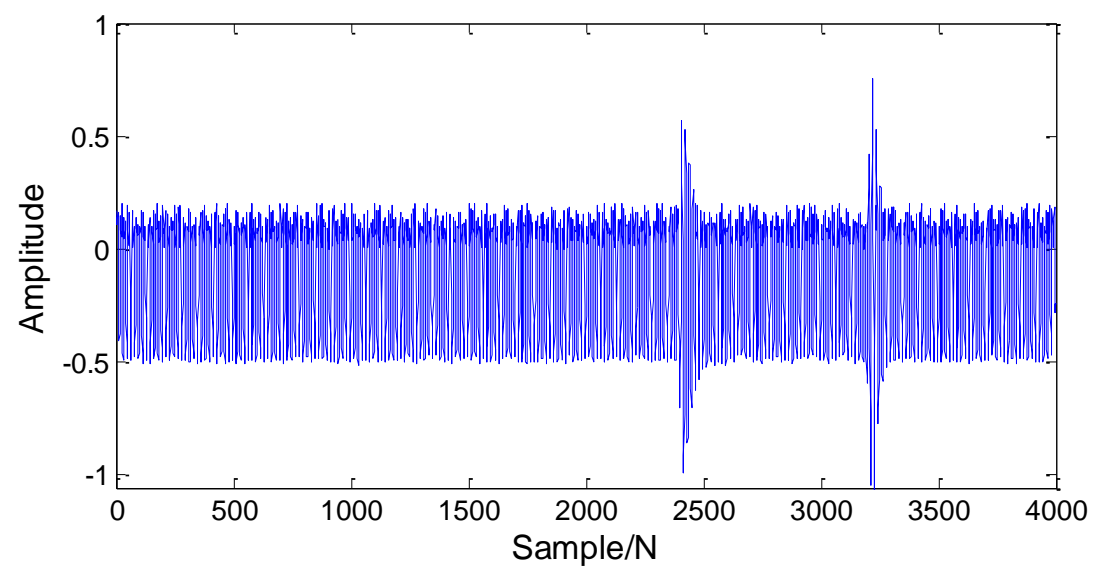

(c)

Figure 6. Comparison of PD signal extraction results: (a) Proposed CEEMDAN-Hankel-SVD method; and (b) Complete ensemble empirical mode decomposition (CEEMD)-based method; and (c) Variation mode decomposition (VMD) wavelet threshold method. 
Table 1. Denoising effect evaluation parameters of each method.

\begin{tabular}{cccc}
\hline Evaluation Parameter & Signal Extraction Method & Pulse 1 & Pulse 2 \\
\hline \multirow{2}{*}{ SNR } & Method of this paper & 16.2860 & 14.9465 \\
& CEEMD & 1.3714 & 7.7682 \\
& VMD wavelet threshold method & 1.8403 & 3.7937 \\
\hline \multirow{2}{*}{ NCC } & Method of this paper & 0.9927 & 0.9940 \\
& CEEMD & 0.8313 & 0.5842 \\
& VMD wavelet threshold method & 0.6551 & 0.8328 \\
\hline \multirow{2}{*}{ VTP } & Method of this paper & 0.9787 & 1.0134 \\
& CEEMD & 1.4194 & 1.1698 \\
& VMD wavelet threshold method & 0.7166 & 0.7888 \\
\hline \multirow{3}{*}{ Amplitude relative error (\%) } & Method of this paper & 0.8 & 1.68 \\
& CEEMD & 32.68 & 11.53 \\
& VMD wavelet threshold method & 7.95 & 14.90 \\
\hline
\end{tabular}

According to a comparison of the extraction results shown in Figure 6 and the indicator values listed in Table 1, the following conclusions can be drawn:

1 The proposed CEEMDAN-Hankel-SVD-based PD signal extraction method provides a high SNR after extraction with no significant distortion in the waveform and low energy loss.

2 The CEEMD-based extraction method suppresses the noise in the signal well. However, the loss of signal energy is excessive.

3 The VMD wavelet threshold method is consistent with the value of $f_{c}$ adopted in the PD signal. Therefore, the periodic narrowband interference cannot be filtered out. Consequently, this method fails to achieve a good signal extraction effect.

Figure 7a,b present detailed waveform comparisons of the ideal noise-free simulated PD signal based on (16) and simulated the PD signal based on (17) in Figure 3a and the corresponding signals extracted from the noisy signal in Figure $3 b$ by the CEEMDAN-Hankel-SVD method (which are reproduced here from Figure 6). The results clearly indicate that the CEEMDAN-Hankel-SVD-based signal extraction algorithm suppressed the periodic narrowband noise and white noise in the noisy PD signal and obtained an extracted PD signal with very little waveform distortion. This provides an excellent basis for conducting fault localization analysis.

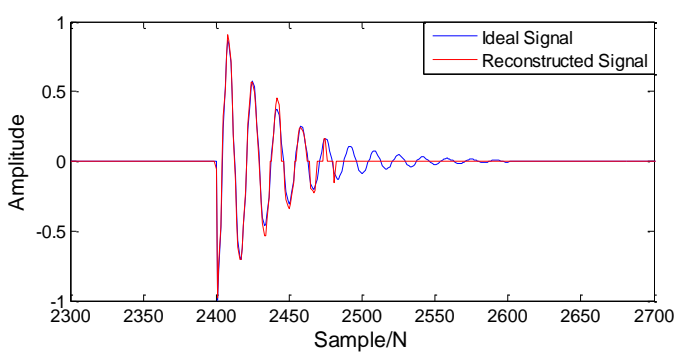

(a)

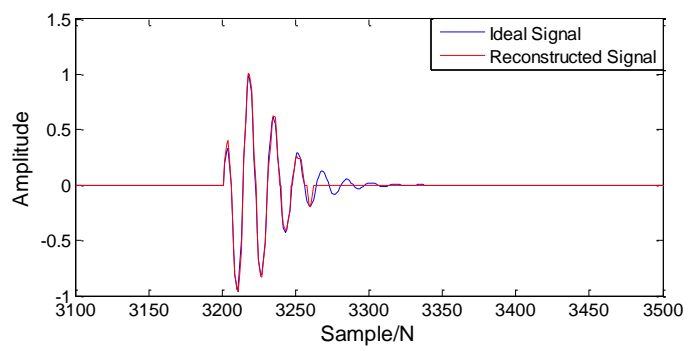

(b)

Figure 7. Detailed waveform comparison between the ideal noise-free PD signals shown in Figure $3 a$ and the signals extracted from the noisy signal in Figure $3 b$ by the CEEMDAN-Hankel-SVD method (Figure 6): (a) Simulated signal based on (16); and (b) Simulated signal based on (17). Here, the figures were placed in separate figures to facilitate detailed comparison.

\subsection{Stability Analysis of Algorithm for White Noise}

The simulation experiments of Section 5.2 processed narrowband signals of different amplitudes and frequencies. In order to quantitatively study the effectiveness of the proposed method on white 
noise interference, white noise with a variance of 0.2 to 0.7 was added based on the original narrowband interference. The curve of the four evaluation indicators is shown in Figure 8.

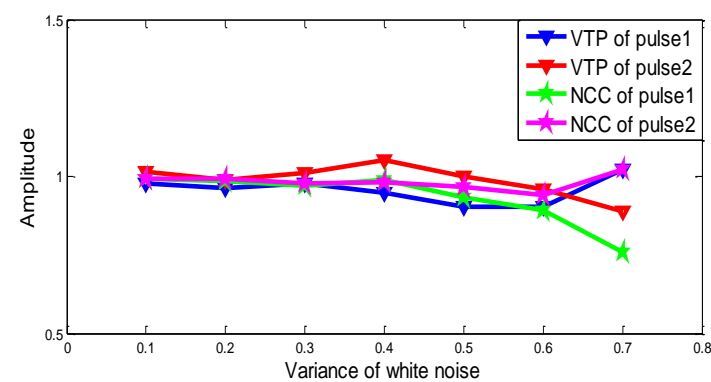

(a)

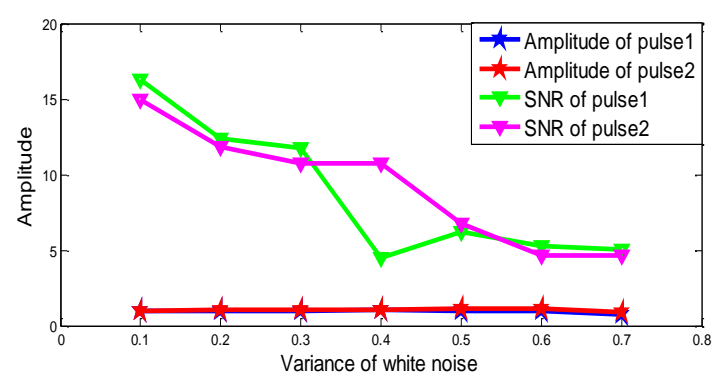

(b)

Figure 8. CEEMDAN-Henkel-SVD denoising results under different white noise variances: (a) The waveform of normalized correlation coefficient (NCC) and variation trend parameter (VTP) after denoising; and (b) The waveform of amplitude and SNR after denoising.

Figure 8 shows that when the white noise variance was $0.1,0.2$, and 0.3 , the four indexes of the two pulses of the PD signal after denoising had a good robustness. When the white noise variance increased up to 0.4, the VTP of the PD pulse was shifted and the SNR of the pulse dropped sharply. This is because after the denoising, the slope of the rising or falling edge of the pulse became larger, resulting in a decrease in the VTP parameter. In addition, in the signal-to-noise ratio calculation method used herein, the maximum value of the amplitude difference before and after the pulse denoising is regarded as the amplitude of the noise, and therefore, the SNR was lowered due to the change in the slope of the rising or falling edge of the pulse. When the white noise variance was 0.7 , the NCC and VTP offsets were severe after denoising and the waveform was distorted after denoising.

\subsection{Measured PD Pulse Detection for Arrival Time Assessment on Different SNR}

In the cable partial discharge localization algorithm, the existence of noise is an important factor affecting the positioning accuracy [9-12]. In order to further verify the practicability of the proposed algorithm, the time-varying kurtosis method [29] was used to pick up the time when the partial PD pulse reached the sensor under high background noise. In fact, in the positioning algorithm based on the HFCT, the pulse arrival time was the key to improving the positioning accuracy. In this paper, the partial discharge signal was collected by the experimental circuit shown in Figure 9a and the HFCT was sleeved on the grounded copper wire. The sampling frequency of the oscilloscope was $100 \mathrm{MHz}$ and the partial discharge measured signal was obtained, as shown in Figure 9b. In order to facilitate subsequent processing, the measured signal amplitude was normalized. Because the laboratory was relatively closed, the measured signal noise content was low. After artificially adding noise (consistent with the noise in the simulated signal), the algorithm was used to denoise the measured signal, and the denoising result is shown in Figure 9d. Then, the time-varying kurtosis method was used to pick up the arrival time of the reconstructed signal, and its time-varying kurtosis curve is shown in Figure 9e. As can be seen from Figure 9e, the maximum value of the time-varying kurtosis coincided with the arrival time of the manually picked-up PD, which is the 262nd sampling point. 


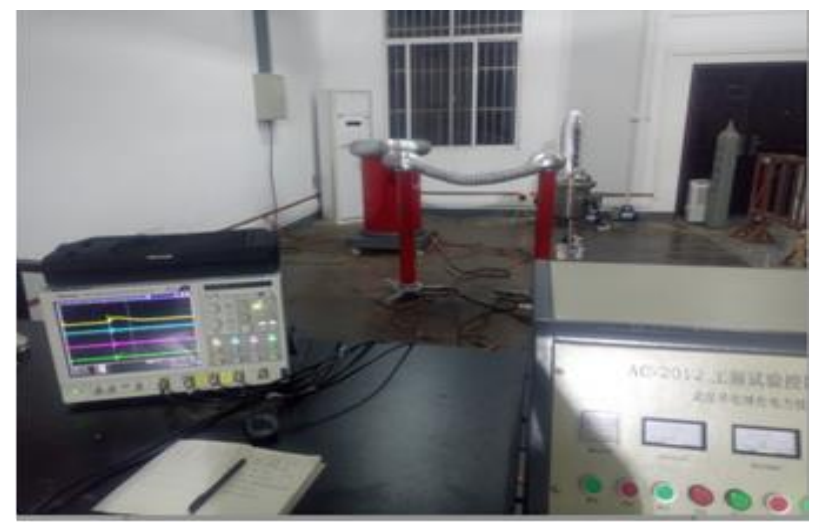

(a)

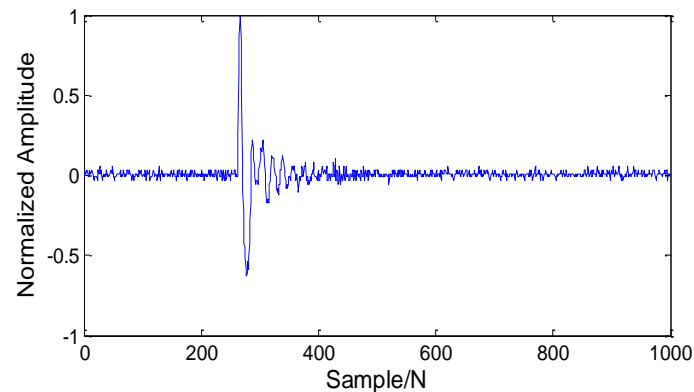

(b)

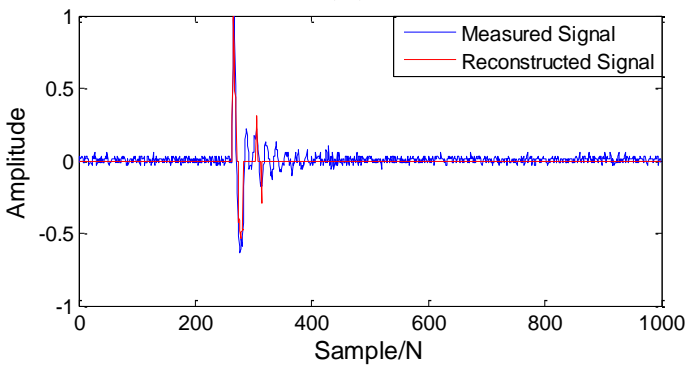

(d)

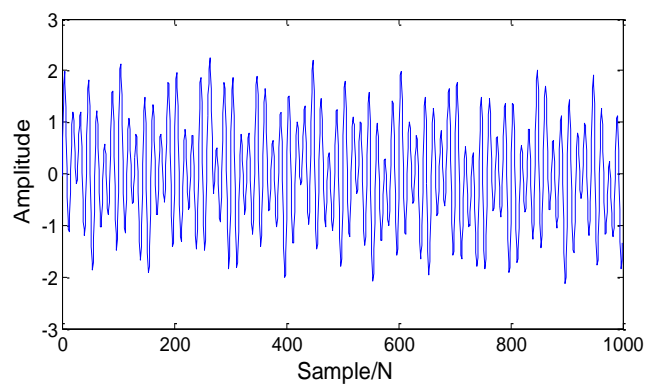

(c)

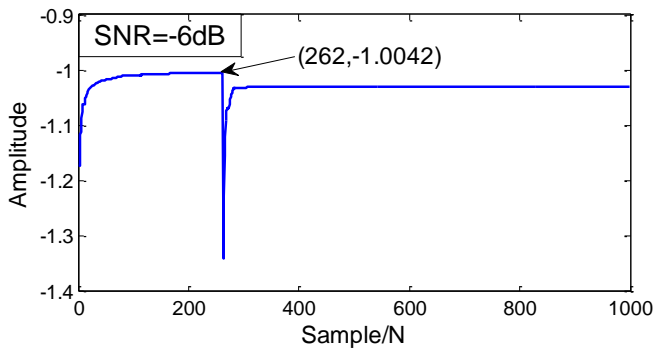

(e)

Figure 9. Measured data processing result: (a) PD experiment platform; (b) Measured PD signal; (c) Measured PD signal after artificially adding noise; (d) The result of denoising by the algorithm of this paper; and (e) Time-varying kurtosis curve of the PD signal shown in Figure 8d.

The impact of adding different high noise levels to the PD signal in Figure $9 \mathrm{~b}$ on the arrival times obtained by the time-varying kurtosis method when applied to the signal extracted by the proposed CEEMDAN-Hankel-SVD method was also considered to further demonstrate the performance of the proposed approach. Therefore, we added Gaussian white noise and periodic narrowband noise with equivalent characteristics to those discussed above, but with variances and amplitudes appropriately scaled to obtain SNR values of $-9,-14$, and $-23 \mathrm{~dB}$, and the resulting noise-containing PD signal and its corresponding time-varying kurtosis curve obtained from the extracted PD signal are shown in Figure 10. Since the measured PD signal without noise was not available, the four indicators for evaluating the denoising result in Section 5.2 were no longer applicable. For this reason, the noise suppression ratio $\rho_{\mathrm{NRR}}$ was introduced [30], and the larger the value, the better the noise suppression effect. In addition, the arrival times obtained from the time-varying kurtosis curves and $\rho_{\text {NRR }}$ are listed in Table 2. 


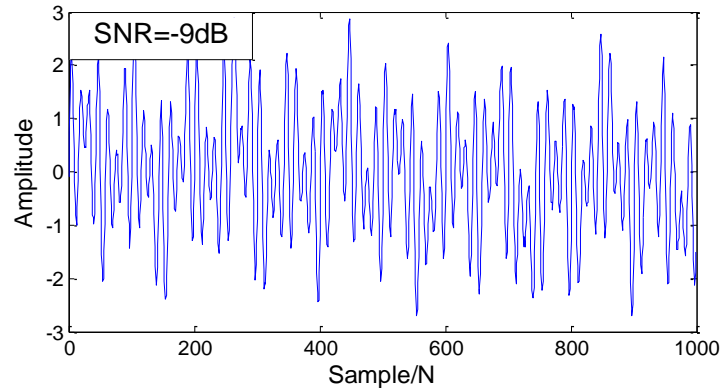

(a)

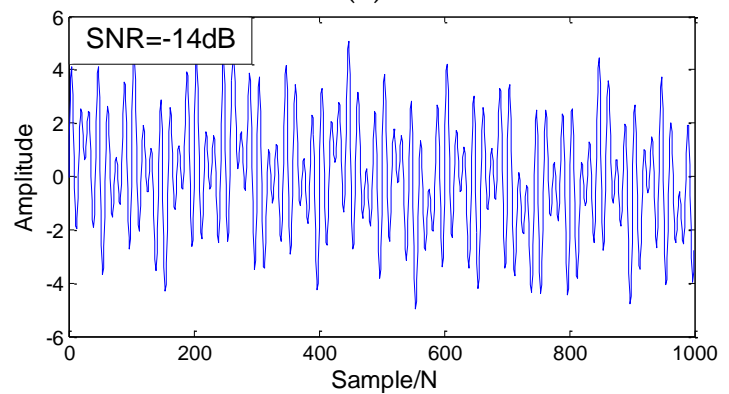

(c)

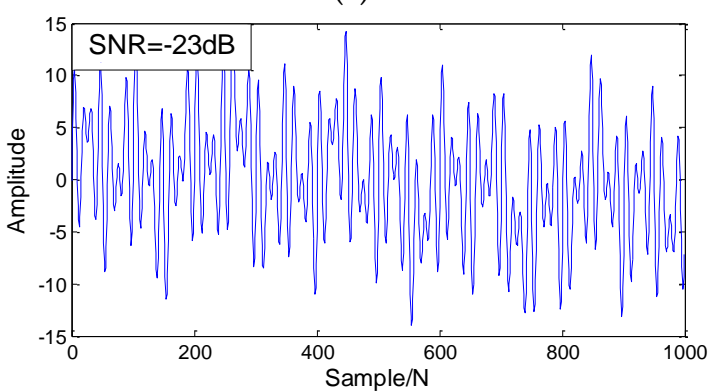

(e)

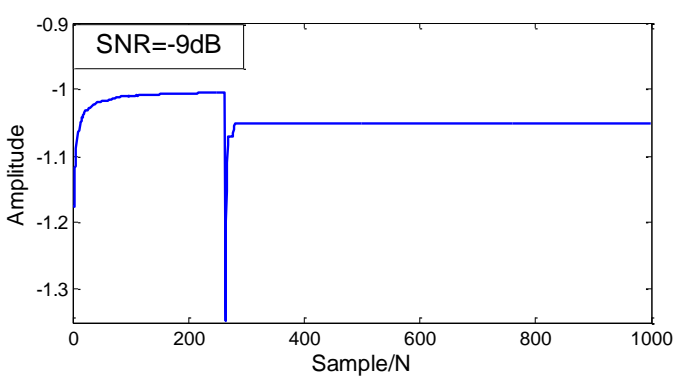

(b)

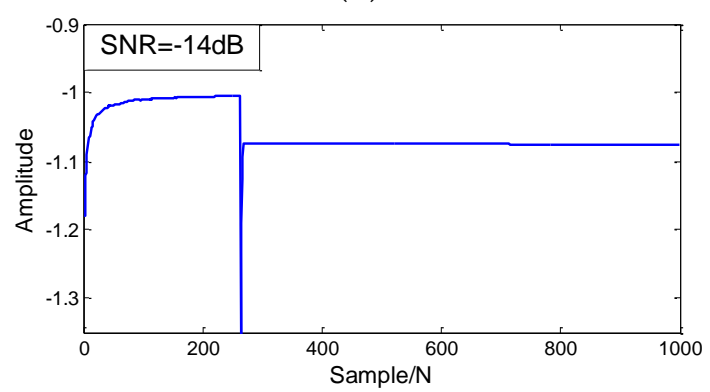

(d)

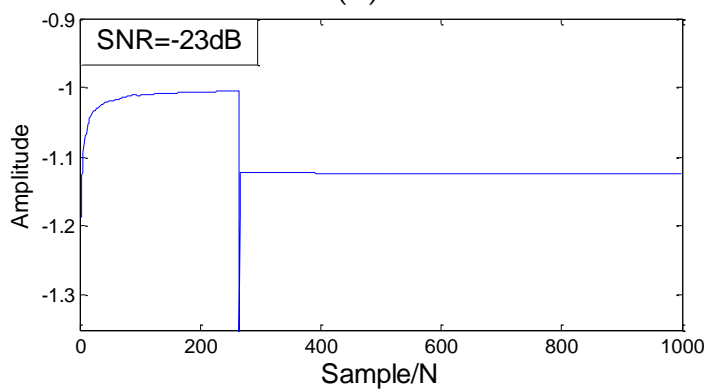

(f)

Figure 10. Noisy PD signals with varying SNR values based on the signal in Figure 8b: (a,c,e); Corresponding time-varying kurtosis curves of the PD signals extracted from the noisy PD signals using the proposed CEEMDAN-Hankel-SVD method: $(\mathbf{b}, \mathbf{d}, \mathbf{f})$.

Table 2. The number of PD signals picked up by different signal-to-noise ratios.

\begin{tabular}{cccccc}
\hline SNR & $\mathbf{- 6} \mathbf{~ d B}$ & $\mathbf{- 9} \mathbf{~ d B}$ & $\mathbf{- 1 4} \mathbf{~ d B}$ & $\mathbf{- 2 3 ~ d B}$ & Manual Picking \\
\hline Point & 262 & 263 & 263 & 263 & 262 \\
\hline$\rho_{\text {NRR }}$ & 88.8097 & 98.4420 & 116.9203 & 148.3636 & - \\
\hline
\end{tabular}

As Table 2 demonstrates, the arrival time obtained from the time-varying kurtosis curve when the SNR was $-6 \mathrm{~dB}$ was consistent with the arrival time obtained by the visual inspection of the waveform. However, when the SNR was $-9 \mathrm{~dB}$, the multiple number of arrival times obtained began to produce arrival time errors, while the arrival time error remained fairly constant as the SNR continued to decrease. These errors were largely due to the use of the wavelet packet threshold method for suppressing the majority of white noise in the PD signals because the waveform information of the relatively low amplitude PD signals was gradually lost as the number of decomposition layers increased. This was the main reason for the pick-up error of the PD signal at the first arrival time. However, the localization accuracy obtained from a PD signal pulse can be controlled within a certain limited range, provided the maximum amplitude of the PD signal after denoising is picked up correctly and is accurately extracted. 


\section{Conclusions}

This paper proposed a PD signal extraction method denoted as the CEEMDAN-Hankel-SVD algorithm that combined CEEMDAN and SVD based on the Hankel matrix formulation to suppress periodic narrowband noise. Then, the remaining white noise was suppressed using an improved wavelet packet threshold method. The performance of the proposed algorithm was demonstrated by its application to synthesized PD signals with complex noise environments composed of both Gaussian white noise and periodic narrowband noise. The results of this paper support the following conclusions:

1 The CEEMDAN-Hankel-SVD algorithm suppresses frequency aliasing and periodic narrowband interference in synthesized PD signals.

2 Compared with the VMD wavelet threshold method, the CEEMDAN-Hankel-SVD method has no frequency limitations for the periodic narrowband noise that can be filtered out, and noisy PD signals can be adaptively decomposed.

3 The threshold function employed in the improved wavelet packet threshold method combines the advantages of soft and hard thresholds and not only accurately preserves the continuity of the PD signal, but also reduces the constant deviation between the wavelet coefficient of the noisy signal and the estimated noise-free wavelet coefficient. Accordingly, the noise-free PD signal was extracted with high fidelity. Therefore, the proposed approach lays a good foundation for conducting subsequent work on signal pattern recognition and fault localization.

4 The time-varying kurtosis method obtains highly accurate arrival times when applied to PD signals extracted by the proposed CEEMDAN-Hankel-SVD method from synthesized signals in complex noise environments with low SNR values.

Author Contributions: K.S. and J.Z. provided the basic ideas of this paper, and carried out experiments and analysis; J.Z., W.S. and J.G. carried out algorithm programming; the manuscript was written by K.S. and J.Z. together; J.Z. revised and improved the manuscript.

Funding: This research was funded by National Natural Science Foundation of China, Grant No. 61403130.

Conflicts of Interest: The authors declare no conflict of interest.

\section{References}

1. Gao, C.; Yu, Y.; Wang, Z.; Wang, W.; Zheng, L.; Du, J. Study on the Relationship between Electrical Tree Development and Partial Discharge of XLPE Cables. J. Nanomater. 2019, 2019, 8932312. [CrossRef]

2. Florkowski, M.; Florkowska, B.; Zydron, P. Partial Discharges in Insulating Systems of Low Voltage Electric Motors Fed by Power Electronics-Twisted-Pair Samples Evaluation. Energies 2019, 12, 768. [CrossRef]

3. Jiang, J.; Zhao, M.; Zhang, C.; Chen, M.; Liu, H.; Albarracin, R. Partial Discharge Analysis in High-Frequency Transformer Based on High-Frequency Current Transduce. Energies 2018, 11, 1997. [CrossRef]

4. Wang, Y.; Li, X.; Gao, Y.; Zhang, H.; Wang, D.; Jin, B. Partial Discharge Ultrasound Detection Using the Sagnac Interferometer System. Sensors 2018, 18, 1425. [CrossRef] [PubMed]

5. Cap, M.; Drexler, P.; Kaska, M. Comparison of methods for detecting the signal arrival time in TDOA localization method. In Proceedings of the 2016 Progress in Electromagnetic Research Symposium (PIERS), Shanghai, China, 8-11 August 2016; pp. 1813-1816.

6. Azirani, M.A.; Ariannik, M.; Werle, P.; Akbari, A. Optimized Arrival Time Determination of UHF Pulses for Localization of Partial Discharges in Power Transformers. In Proceedings of the 2018 IEEE Conference on Electrical Insulation and Dielectric Phenomena (CEIDP), Cancun, Mexico, 21-24 October 2018; pp. 522-526.

7. Wang, B.; Dong, M.; Ren, M.; Wu, Z.; Ma, A. Partial discharge ultrasonic localization simulation based on double helix ultrasonic sensor array. In Proceedings of the 2018 12th International Conference on the Properties and Applications of Dielectric Materials (ICPADM), Xi'an, China, 20-24 May 2018; pp. 136-139. 
8. Khan, U. Localization of Partial Discharge by Using Received Signal Strength. In Proceedings of the 2018 2nd URSI Atlantic Radio Science Meeting (AT-RASC), Meloneras, Spain, 28 May-1 June 2018; pp. 1-4.

9. Mehdizadeh, S.; Yazdchi, M.; Niroomand, M. A Novel AE Based Algorithm for PD Localizationin Power Transformers. J. Electr. Eng. Technol. 2013, 8, 1487-1496. [CrossRef]

10. Li, P.; Zhou, W.; Yang, S.; Liu, Y.; Tian, Y.; Wang, Y. Method for partial discharge localisation in air-insulatedsubstations. IET Sci. Meas. Technol. 2017, 11, 331-338. [CrossRef]

11. Bai, F.; Gagar, D.; Foote, P.; Zhao, Y. Comparison of alternatives to amplitude thresholding for onset detection of acoustic emission signals. Mech. Syst. Signal Process. 2017, 84, 717-730. [CrossRef]

12. Sun, K.; Guo, J.; Ma, X. On line partial discharge detection and localization based on the wavelet packet-kurtosis. J. Electron. Meas. Instrum. 2017, 31, 1099-1106.

13. Wang, Y.; Li, Q.; Zhou, F.; Zhou, Y.; Mu, X. A New Method with Hilbert Transform and Slip-SVD-Based Noise-Suppression Algorithm for Noisy Power Quality Monitoring. IEEE Trans. Instrum. Meas. 2019, 68, 987-1001. [CrossRef]

14. Liu, S.; Sun, Y.; Ma, W.; Xie, F.; Jiang, X.; He, L.; Kang, Y. A New Signal Processing Method Based on Notch Filtering and Wavelet Denoising in Wire Rope Inspection. J. Nondestruct. Eval. 2019, 38, 39. [CrossRef]

15. Bharathi, B.M.R.; Mohanty, A.R. Time delay estimation in reverberant and low SNR environment by EMD based maximum likelihood method. Measurement 2019, 137, 655-663. [CrossRef]

16. Lei, Y.; He, Z.; Zi, Y. Application of the EEMD method to rotor fault diagnosis of rotating machinery. Mech. Syst. Signal Process. 2009, 23, 1327-1338. [CrossRef]

17. Yeh, J.; Shieh, J.; Huang, N.E. Complementary ensemble empirical mode decomposition: A novel noise enhanced data analysis method. Adv. Adapt. Data Anal. 2010, 2, 135-156. [CrossRef]

18. Altay, Y.A.; Kremlev, A.S. Improved noise resistance processing electrocardiosignals adaptive noise CEEMDAN as a method of solving the basic problems of the application of Hilbert-Huang transform. In Proceedings of the 2017 IEEE Conference of Russian Young Researchers in Electrical and Electronic Engineering (EIConRus), St. Petersburg, Russia, 1-3 February 2017; pp. 621-623.

19. Qian, Y. A wavelet denoising method based on improved threshold and autocorrelation. In Proceedings of the 2018 Chinese Control and Decision Conference (CCDC), Shenyang, China, 9-11 June 2018; pp. 4058-4063.

20. Zhang, C.; Li, X.; Zhang, M. A novel ECG signal denoising method based on Hilbert-Huang Transform. In Proceedings of the 2010 International Conference on Computer and Communication Technologies in Agriculture Engineering, Chengdu, China, 12-13 June 2010; pp. 284-287.

21. Rojas-Moreno, M.V.; Robles, G.; Martínez-Tarifa, J.M.; Fresno, J.M. Ensemble Empirical Mode Decomposition for the denoising of partial discharges measured in UHF. In Proceedings of the 2016 IEEE International Conference on Dielectrics (ICD), Montpellier, France, 3-7 July 2016; pp. 963-966.

22. Zhou, B.; Liu, Z. Method of Multi-resolution and Effective Singular Value Decomposition in Under-determined Blind Source Separation and Its Application to the Fault Diagnosis of Roller Bearing. In Proceedings of the 2015 11th International Conference on Computational Intelligence and Security (CIS), Shenzhen, China, 19-20 December 2015; pp. 462-465.

23. Hen, Y.; Cheng, Y.; Liu, H. Application of improved wavelet adaptive threshold de-noising algorithm in FBG demodulation. Optik 2017, 132, 243-248.

24. Wang, X.; Ou, X.; Chen, B.; Kim, M. Image Denoising Based on Improved Wavelet Threshold Function for Wireless Camera Networks and Transmissions. Int. J. Distrib. Sens. Netw. 2015, 11, 670216. [CrossRef]

25. Luo, H.; Zhang, X.A. Method of Second Wavelet Transform Automated Threshold for Partial Discharge Signal Extraction. In Proceedings of the 2011 Second International Conference on Digital Manufacturing Automation, Zhangjiajie, China, 5-7 August 2011; pp. 42-45.

26. Pan, Y.; Zhang, X.; Zhang, Y. Denoising Method for Transformer Partial Discharge Signals Based on Complete Ensemble Empirical Mode Decomposition. Guangdong Electr. Power 2017, 30, 93-98.

27. Jiao, S.; Shi, W.; Liu, Q. Self-adaptive partial discharge denoising based on variational mode decomposition and wavelet packet transform. In Proceedings of the 2017 Chinese Automation Congress (CAC), Jinan, China, 20-22 October 2017; pp. 7301-7306.

28. Tang, J.; Gao, L.; Peng, L. Study on New Evaluation Parameters for De-noising Performance of Non-stationary Oscillating Partial Discharge Signals. High Volt. Eng. 2007, 12, 66-70. 
29. Sun, K.; Guo, J.; Yang, Y. On Line Partial Discharge Detection and Localization Based on AIC Criterion and Time-Window Energy Ratio. Chin. J. Sens. AK Actuators 2017, 30, 1209-1214.

30. Liu, Y.; Lv, F.; Li, C.; Li, Y. Study of the mathematical morphological filter in suppressing periodic narrow bandwidth noise of PD. Zhong Guo Dian Ji Gong Cheng Xue Bao/Proc. Chin. Soc. Electr. Eng. 2004, 24, 169-173.

(C) 2019 by the authors. Licensee MDPI, Basel, Switzerland. This article is an open access article distributed under the terms and conditions of the Creative Commons Attribution (CC BY) license (http://creativecommons.org/licenses/by/4.0/). 